\title{
A modern take on the theoretical modelling of inertial properties of a human body for biomechanical simulations
}

\author{
William S. P. Robertson ${ }^{\mathrm{a}}$

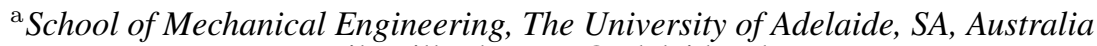 \\ Email: will.robertson@adelaide.edu.au
}

\begin{abstract}
The accurate estimation of inertial properties of individual body segments, including mass and moment of inertia, for biomechanical modelling and simulation is important in predicting realistic movement patterns. These properties must be subject-specific, as body shape and weight distribution varies significantly between members of the human population. One method of estimating these parameters is by using anthropometric measurements as inputs into a geometric humanoid model, in which each body segment is comprised of a certain number of regular volumetric shapes depending on the complexity of the shape and on the complexity of the model.
\end{abstract}

Several of these geometric body segment parameter models have been proposed, among which the work of the late Herbert Hatze (1937-2002) stands out as the most detailed and accurate. Due to the mathematical complexity of his model (1979-1980), his work has not been widely used for biomechanical research; while often cited, it has rarely been reproduced.

In this paper, an open source redevelopment of Hatze's model is described, which includes a visualisation component that allows the 3D geometry to be accurately depicted for the first time. An analysis is performed which relates the accuracy of the model to the number of anthropometric measurements taken and suggests a reduced-order version of the model using interpolation. In order for the model to be more widely used and hopefully improved in time, the Matlab code for this work is made freely available for use by the research community.
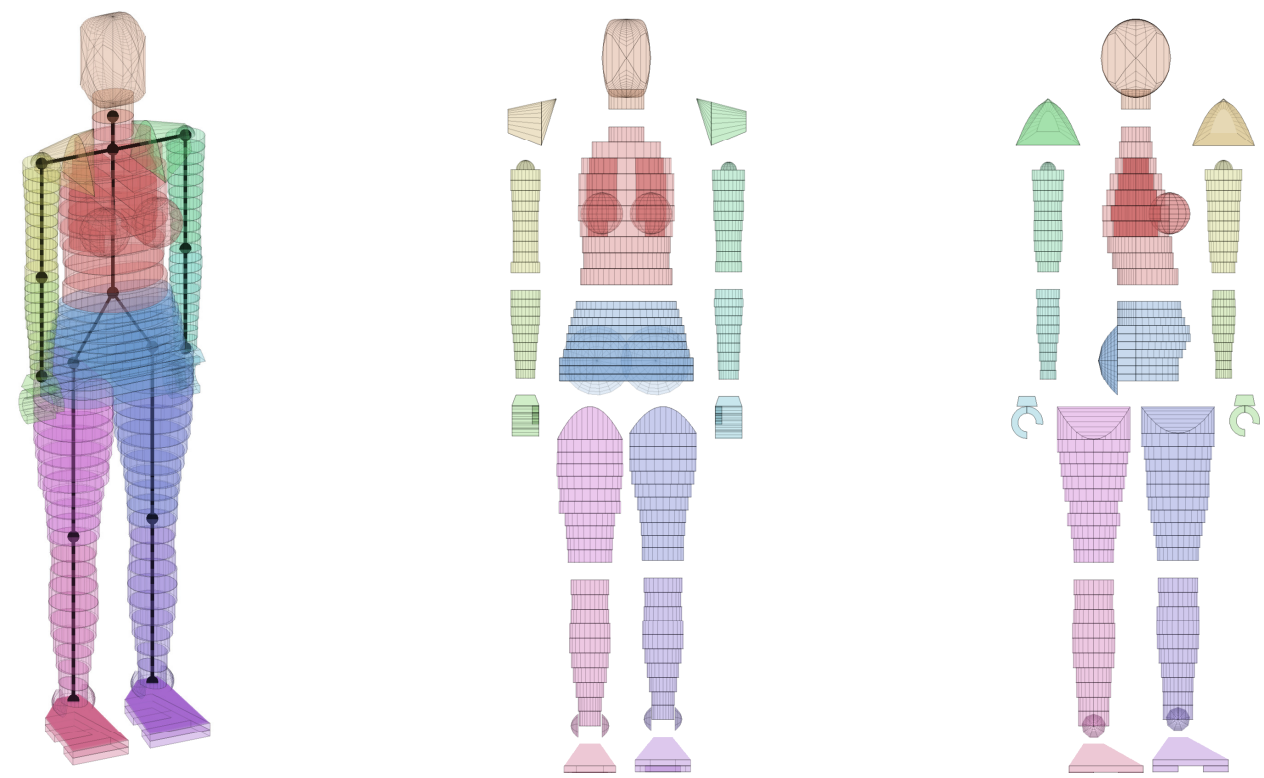

Keywords: Biomechanics, body segment parameter estimation 


\section{INTRODUCTION}

For biomechanical simulations, models are used to estimate inertial properties of each individual body segment of a subject; these properties include mass, centroid, and moment of inertia. The complexity of a body segment parameter model is governed by the number of measurements taken. In this paper, the body segment parameter model of Hatze (1979) is revived having received comparatively little attention from the research community due to its complexity of implementation. As part of this work, the Matlab programme of this model is made freely available (Robertson 2013).

The content of this paper is as follows. Body segment modelling is briefly introduced, including the two main approaches taken in the literature. This is followed by an overview of the model used in this work and a discussion on the difficulties that arose in its reconstruction and reimplementation. A model reduction is proposed to reduce its complexity, with results that indicate the number of measurements taken may be dramatically reduced, and the paper concludes with a discussion of future work for the project.

\section{BODY SEGMENT PARAMETER MODELS}

Two methods are commonly used for estimating body segment parameters: regression equations and geometric approximation. The former technique is based on regression equations derived from cadaveric measurements, computed tomography, plain radiograph, or magnetic resonance imaging, etc., data from a finite set of specimens. This approach can be considered to extrapolate from an average and has little accuracy for modelling an individual that falls outside the proportions of the initial dataset (Pearsall and Reid 1994).

The geometric approximation technique uses in-field measurements of an individual mapped onto a 3D geometric model to derive mass and related properties from certain shape and density assumptions. This technique is limited by the number of measurements that can be practically taken on an individual and the complexity of the geometry used to model their body shape.

\subsection{Regression equation models}

The regression equation technique for estimating body segment parameters is the more popular due to it being simpler to implement and use. For example, the work of Leva (1996) contains tables of data for average male and female segment parameters which can be scaled appropriately according to an individual's height, mass, and segment lengths. For the purposes of this work, these techniques are avoided since regression equations are known to produce inaccurate results over a disparate population of body types (Durkin and Dowling 2003). (Consult such citations for extended literature on body segment parameter modelling using regression equations.)

\subsection{Geometric approximation models}

Hanavan (1964) proposed the earliest geometric model of a humanoid, consisting of fifteen homogeneous solid shapes including ellipsoids, truncated cones, and elliptic cylinders. Twenty-five anthropometric measurements were required to calculate the properties of the model. Jensen (1978) noted the inadequancy of using homogeneous and regular segments in Hanavan's model since segments can be subject to significant shape and density fluctuations along their length. His work proposed $2 \mathrm{~cm}$ elliptical slices of the human body measured using photometric means; the accuracy of estimating segment masses was significantly improved over the method of Hanavan.

Hatze (1979) developed a remarkably detailed seventeen segment 3D geometrical model of the human body with the same motivation, but due to its complexity this model has rarely been used by other researchers in the field. Previous work by Hanavan (1964) is simple but inaccurate, while the work of Yeadon (1990) using the general 'stadium solid' shape forms a middle ground between ease of modelling and accuracy. A comparison by Wicke, Dumas, and Costigan (2009) between the regression equation technique and geometric modelling (although not including the work of Hatze) showed that the more complex geometric models produce more accurate results, especially when non-uniform densities are used; although Hatze's model has its flaws it is the most complete geometric model yet developed.

\section{OVERVIEW OF HATZE'S MODEL}

In the models by Hanavan (1964) and Yeadon (1990), segments are comprised of one (for Hanavan) or a small number ( 2 for the limbs, 4 for the torso, for Yeadon) of geometric shapes of constant density. By contrast, 


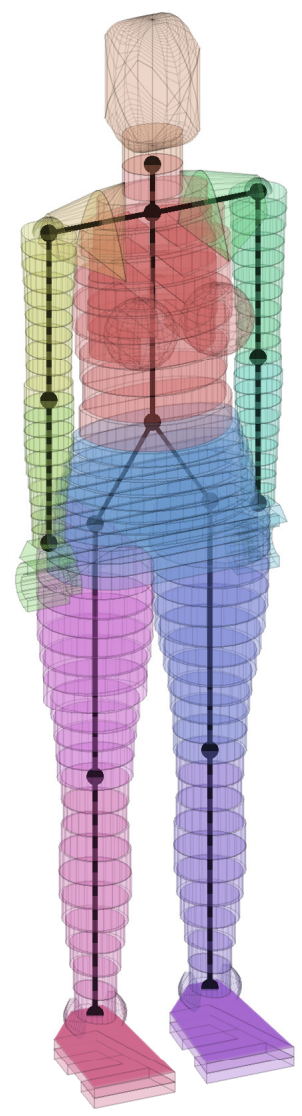

ISOMETRIC

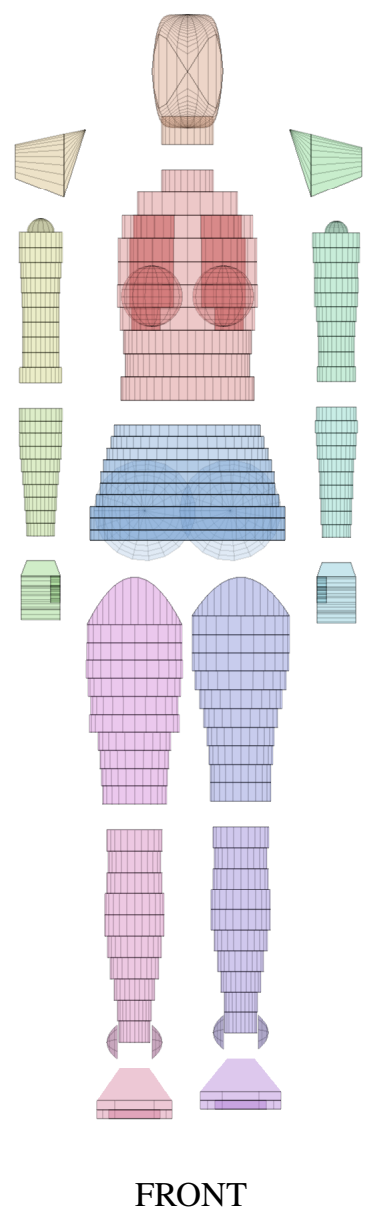

Figure 1. The seventeen segments of Hatze's model, aligned by joint centre in the isometric view and separated for clarity in the orthogonal views. The female variation is displayed.

Hatze's model breaks almost every body segment into a large number of slices or 'plates', each with specific sizes and densities (Figure 1). Hatze's model is also distinguished by the use of a large number of different geometric shapes, which are:

- elliptic plates (arms, legs, neck),

- semi-elliptic plates (thoracic and pelvic regions),

- trapezoidal plates (pelvic region, feet),

- elliptic paraboloids (ankles, buttocks),

- parabolic plates (lungs),

- parabolic 'wedges' (shoulders),
- hemispheres (humeral heads, breasts),

- hollow cylindrical sections (fingers, thumb),

- rectangular prisms (hands), and

- rectangular prisms with rounded edges (pelvic region).

Each of these shapes require various measurements to calculate their extents, such as diameter, perimeter, and length. The total number of measurements required for Hatze's model is 242, with a full 160 of these being diameters and perimeters of ellipses for modelling the limbs; it is interesting to consider the effect of taking a smaller number of measurements on the accuracy and convenience convenience of applying the model. Before discussing the possibility of such modifications to Hatze's work, a summary of the work required to implement his model will be presented.

\section{RE-IMPLEMENTING HATZE'S MODEL}

Hatze's work, to the author's knowledge, has not been reproduced in full by the biomechanics community. This is presumably due to its complexity. First of all, the paper which describes the model (Hatze 1980) does not include the mathematical details of the model itself due to space constraints. We obtained a copy of the 
technical report with these details (Hatze 1979) directly from the Council for Scientific and Industrial Research, Pretoria, South Africa.

Even with the mathematical treatment of the model, however, there are still unanswered questions about the construction of the model and its verification. The greatest difficulty in reproducing the work is that the tabulated example measurements (provided in the appendix) are not labelled, and in many cases the correspondence between measured values and physical dimensions is ambiguous.

While Hatze's own work (Hatze 1981; Hatze 1995) uses an artistic impression of his model, his visual depiction does not have exact verisimilitude with the details of his model. For the purposes of understanding and accurately representing the model, it is necessary to produce a visual representation of its various geometry. Despite the mathematical rigour of Hatze's model, that there are certain details missing from the report hinder its reconstruction. In some cases, several intrinsic dimensions of the body segments that are calculated from measurements are not explained or labelled and must be inferred from the equations given for the centroid and moment of inertia calculations. Two examples of this are described following.

\subsection{Shoulder geometry}

The first example is the presentation of the geometry of the complex shapes used for the shoulder segments. The outer volumes of these segments are constructed using two prisms with parabolic cross section. Hatze's report gives the integrals required to calculate their volume but does not list the geometry explicitly. Due to the large number of coupled variables in this segment, it is somewhat tedious to reverse engineer his shapes. Using Hatze's notation (with additional auxiliary variables $l_{1}, d_{x}, d_{z}$ ), the geometry of the shoulder can be described using measured variables described in Table 1 with calculated variables as follows:

$$
\begin{aligned}
& h_{1}=0.68 a_{t_{5}}-a_{t_{1}}, \quad d_{z}=0.2 l_{t}-z_{h}-1.5 b_{1}, \quad l_{1}=0.35 l_{t}-z_{h}, \\
& \beta=\arcsin \left(d_{z} / d\right), \quad \alpha=\arctan \left(h_{1}, l_{1}\right), \quad d_{x}=d \cos (\beta), \\
& h^{\prime}=d_{x}-h_{1}, \quad \gamma=\arctan \left(l_{1}-2.5 b_{1}-d_{z}, h^{\prime}\right) .
\end{aligned}
$$

From these definitions given by Hatze, it is possible to reconstruct the geometry of the shoulder, which is shown in Figure 2.

\subsection{Abdomino-pelvic geometry}

The abdomino-pelvic region of Hatze's model is the most complex in terms of the variety of shapes used and their careful placement. As partially seen in Figure 1, it consists of two elliptic paraboloids for the buttocks, three posterior and seven anterior semi-elliptic plates for the lower back and abdomen, seven trapezoidal plates for the posterior region beneath the buttocks, and what Hatze describes as three plates of 'special shape' in the anterior pelvic region.

While Hatze gives the equations to calculate volume, etc., for these 'special shapes', he does not describe them and it is left to the reader to infer their shape. Hatze's equation for the volume of the three special shapes is

$$
3 v_{l}=0.3 l\left[2 a_{8} r-a_{t_{1}} b_{t_{1}}[2-\pi / 2]\right],
$$

where $0.3 l$ is the height of the three plates, $a_{8}$ is the half-width of the (top) plate, $a_{t_{1}}, b_{t_{1}}$ are the axes of the first elliptic plate of the thigh, and $r-b_{t_{1}}$ is (inferred to be) the anterior-posterior distance between the origin of the pelvic region and the origins of the thighs. This can be seen to be the volume of a rectangular prism with two inverse-elliptic sections removed as shown in Figure 3 such that the 'special shape' is an elliptically-rounded rectangular prism that follows the outer shape of the top of the thighs. This shape (and hence the position of the origins of the thighs) can be confirmed by analysing Hatze's equation for the $y$-centroid of the special shape ( $\bar{y}_{4}$ in his Eq. A2.82) and the moment of inertia around the $y$ axis (in his Eq. A2.84).

\section{REDUCING THE MODEL}

Because Hatze's model uses elliptic plates for the limbs, a reasonably large number of measurements is required to avoid errors from the stepwise changes in diameter. The model of Yeadon (1990) avoids this problem by using truncated conic sections, but his model assumes that the limbs have circular cross-section, which is an oversimplification. An alternative method to improve the accuracy of the modelling between slices is to interpolate the measurements and use a larger number of slices in the calculation of the segment parameters (Figure 4). 
W. S. P. Robertson, Theoretical modelling of inertial properties of a human body for biomechanical simulations

\begin{tabular}{cl}
\hline Variable & Description \\
\hline$l_{t}$ & Height of abdominal-thoracic segment \\
$a_{t_{1}}$ & Semi-major axis (medial-laterial) of first thoracic plate \\
$a_{t_{5}}$ & Semi-major axis (medial-laterial) of fifth thoracic plate \\
$b_{1}$ & Semi-major axis (medial-laterial) of first arm plate \\
$z_{h}$ & Vertical distance between bottom of neck and top of shoulder \\
$d$ & Lengthwise distance across the top of the shoulder \\
\hline
\end{tabular}

Table 1. Measured variables for determining the shoulder segment geometry.

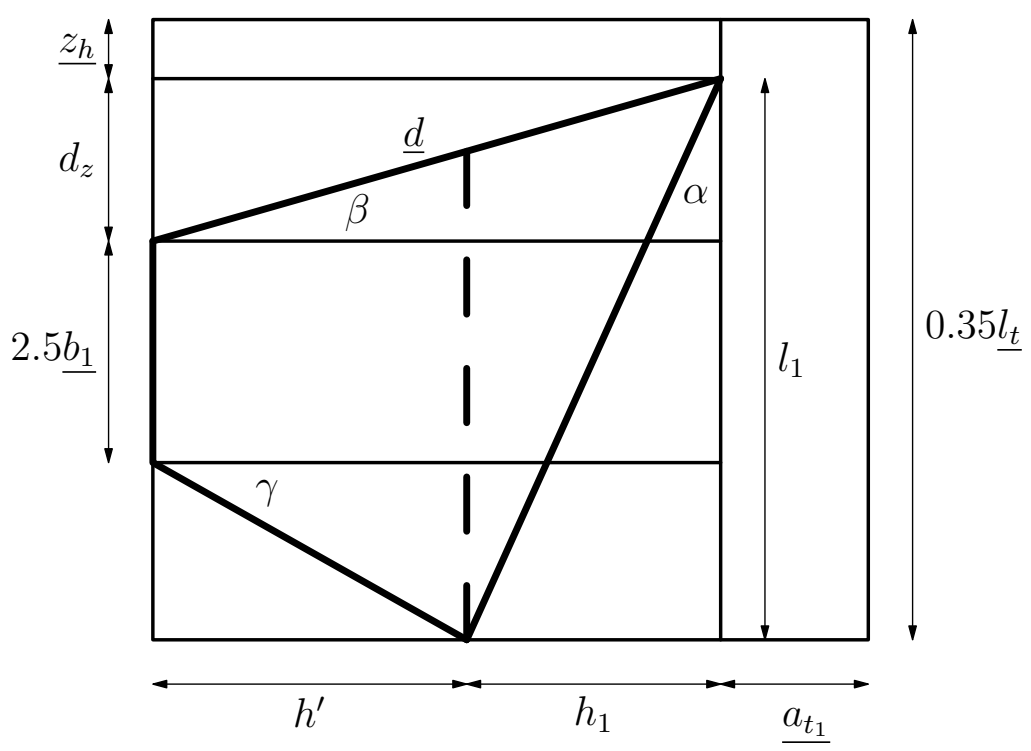

Figure 2. Geometry of the shoulder, front view. The thick lines are the shoulder outline, and the dashed line is the boundary between the two subsegments of the shoulder. Directly measured variables are underlined.

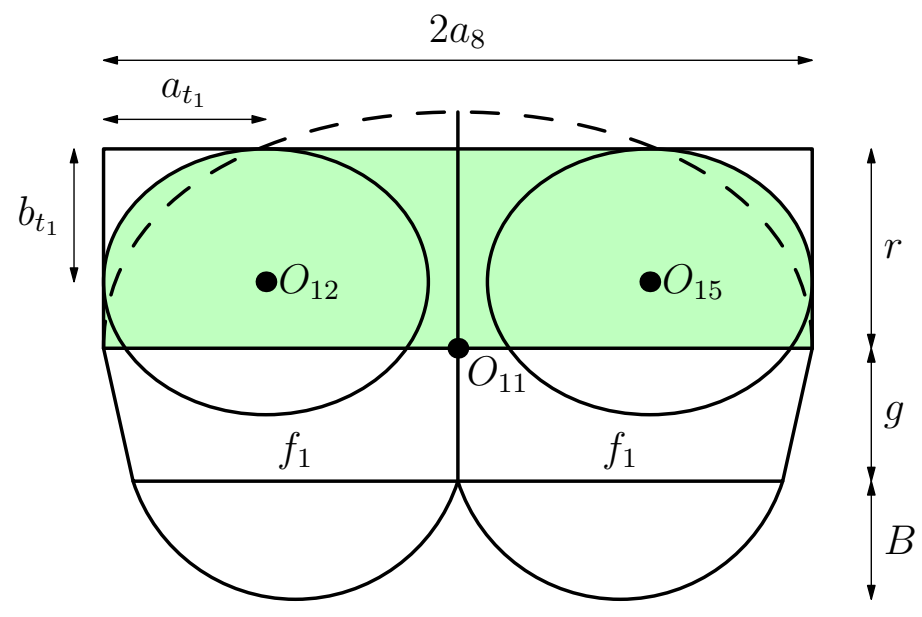

Figure 3. Geometry of the 'special shape' of the anterior inferior pelvic region, top view. The shaded region is the cross-section of the plate of special shape, which can be seen to follow the elliptic shape of the legs (origins $O_{12}$ and $O_{15}$ ). The dashed line is the outline of the semi-elliptic plate superior to the special shape. Notation follows that of Hatze (1979). 
W. S. P. Robertson, Theoretical modelling of inertial properties of a human body for biomechanical simulations

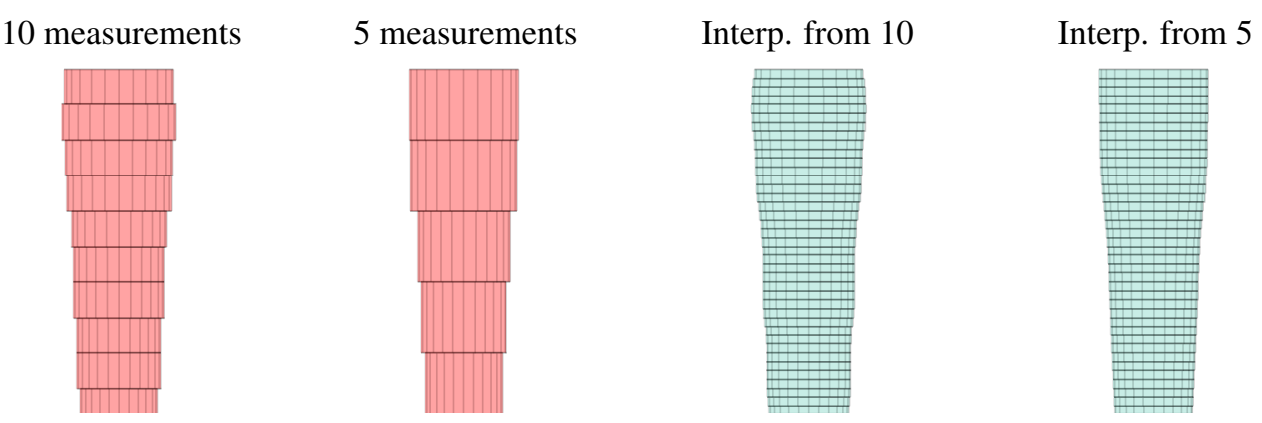

Figure 4. Divisions of the forearm segment. Original (measured) data and halved data are shown in red, with blue segments showing 40 interpolated sub-divisions of each, respectively.

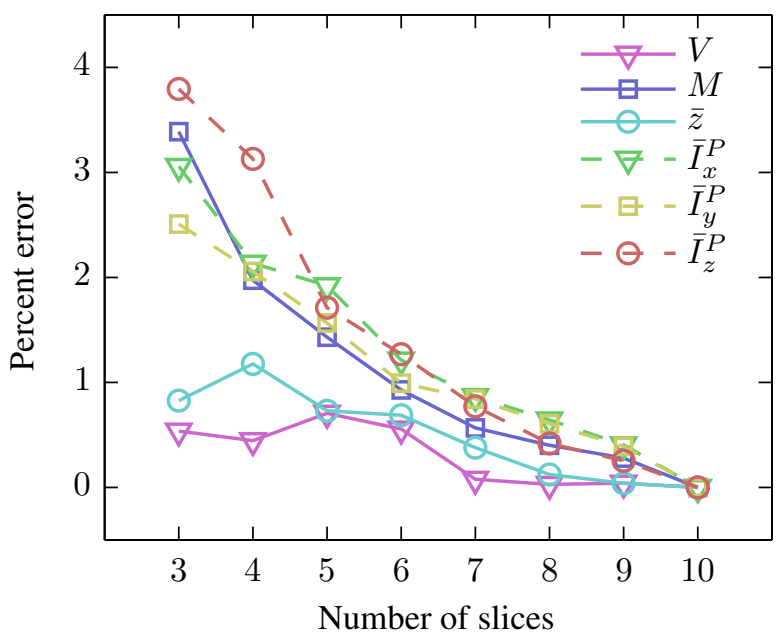

Figure 5. Percent error in the right forearm from reducing the number of measurements taken along the length of the segment.

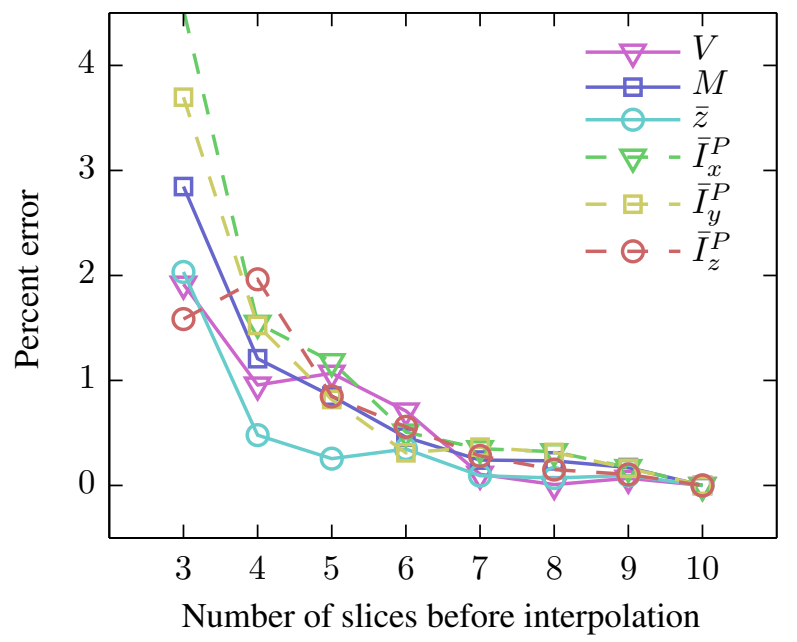

Figure 6. Percent error from taking fewer measurements and interpolating to a larger number of slices.

For this work, an anatomically correct set of reference measurements is not available but the efficacy of this method can still be demonstrated by downsampling Hatze's reference measurements using cubic interpolation to emulate taking a smaller number of anthropometric measurements. The right forearm is chosen for this demonstration. Calculation of the segment parameters, shown in Figure 5, indicates that the error in estimations can rise to approximately $2 \%$ with half the number of measurements ( 5 instead of 10 along the forearm). While this error may seem small, Hatze's model is intended to be accurate to within $3 \%$ overall; increasing the error in each limb parameter by such an amount could reduce the fidelity of the model.

Rather than simply reducing the number of slices (shown in red in Figure 4), better results are obtained if the measurements are interpolated before Hatze's model is applied. That is, while 10 or perhaps fewer measurements are taken, a greater number of slices may be used to represent the segment for the calculations (shown in blue in Figure 4). This results in a reduction of the error from halving the number of measurements (Figure 6) to around $1 \%$. It should be noted that in the absence of higher fidelity reference data, the interpolation used here simply re-increases the number of slices to 10 ; it is expected that improved results will be seen from measuring a larger number of slices, such as with a photographic technique. The results shown here for the forearm are typical of each limb. 


\section{CONCLUSION AND FUTURE WORK}

The work described in this paper is the beginning of a project to provide a freely-available body segment parameter model for the research community. The motivation for this work is due to the complexity of the model; it is non-trivial to reproduce Hatze's work. The program currently available calculates many of the body segment parameters and work is ongoing to verify each set of calculations. Furthermore, an important graphical component is provided that allows Hatze's model to be accurately visualised for the first time.

A model reduction has been proposed that involves taking fewer measurements and interpolating between them to effectively increase the number of slices used in each segment. This technique shows promise and will be verified against anatomical data sets in future work.

The redevelopment of Hatze's model is ongoing. The anthropometric model itself, despite its overall complexity, lacks detail in certain regions, especially the hands and feet. These are areas where additions or adjustments can be made.

Models by other researchers should also be included with the Matlab code to allow for direct comparison; it is possible that the regression equation approach could be fused with the geometric approach to achieve more accurate results over a wider set of the human population. Finally, the visualisation routine currently does not include volumes of 'negative space', such as the cutouts in the shoulders and the pelvic region; these should be incorporated into the generation of the $3 \mathrm{D}$ volumes.

\section{ACKNOWLEDGeMENTS}

Thanks to Jianan (Tony) Wang for his assistance in transcribing Hatze's equations.

\section{REFERENCES}

Durkin, Jennifer L. and James J. Dowling (2003). “Analysis of Body Segment Parameter Differences Between Four Human Populations and the Estimation Errors of Four Popular Mathematical Models". Journal of Biomechanical Engineering 125.4, pp. 515-522. URL: http: / / doi . org/b3m 4 sm.

Hanavan Jr, Ernest P. (1964). A mathematical model of the human body. AMRL Technical Report (TR-64-102). Wright-Patterson Air Force Aerospace Medical Research Lab. URL: http: / / www . dtic.mil/docs / citations/AD0608463.

Hatze, H. (1979). A model for the computational determination of parameter values of anthropomorphic segments. Techn. Report TWISK 79. Pretoria: CSIR.

- (1980). "A mathematical model for the computational determination of parameter values of anthropomorphic segments”. Journal of Biomechanics 13.10, pp. 833-843. URL: http: / / doi . org / d 49 qv.

- (1981). "A comprehensive model for human motion simulation and its application to the take-off phase of the long jump". Journal of Biomechanics 14.3, pp. 135-142. URL: http: / / doi . org / chdt 23.

- (1995). "The extended transentropy function as a useful quantifier of human motion variability". Medicine and science in sports and exercise 27.5, pp. 751-759.

Jensen, Robert K. (1978). "Estimation of the biomechanical properties of three body types using a photogrammetric method". Journal of Biomechanics 11.8-9, pp. 349-358. URL: http: / / doi . org / c 9k2xk.

Leva, Paolo de (1996). “Adjustments to Zatsiorsky-Seluyanov's segment inertia parameters”. Journal of Biomechanics 29.9, pp. 1223-1230. URL: http: / / doi .org/d9h976.

Pearsall, David J. and Gavin Reid (1994). "The Study of Human Body Segment Parameters in Biomechanics". Sports Medicine 18.2, pp. 126-140. URL: http: / / doi .org/dszp36.

Robertson, Will (2013). A Matlab implementation of Hatze's 1980 anthropometric body segment parameter model. Code repository. URL: http://github. com/wspr/hatze-biomech.

Wicke, Jason, Genevieve A. Dumas, and Patrick A. Costigan (2009). "A comparison between a new model and current models for estimating trunk segment inertial parameters". Journal of Biomechanics 42.1, pp. 55-60. URL: http: / / doi .org/d59b69.

Yeadon, M.R. (1990). "The simulation of aerial movement-II. A mathematical inertia model of the human body". Journal of Biomechanics 23.1, pp. 67-74. URL: http: / / doi . org / cgb 472. 